FERMILAB-TM-2054

\title{
Observation of Stimulated Raman Scattering in an Optical Fiber at the Fermilab A0 Photoinjector
}

\author{
A.R. Fry, B. Taylor, M.J. Fitch and A.C. Melissinos \\ University of Rochester \\ Rochester, New York 14627
}

Fermi National Accelerator Laboratory

P.O. Box 500, Batavia, Illinois 60510

August 1998 


\section{Disclaimer}

This report was prepared as an account of work sponsored by an agency of the United States Government. Neither the United States Government nor any agency thereof, nor any of their employees, makes any warranty, expressed or implied, or assumes any legal liability or responsibility for the accuracy, completeness, or usefulness of any information, apparatus, product, or process disclosed, or represents that its use would not infringe privately owned rights. Reference herein to any specific commercial product, process, or service by trade name, trademark, manufacturer, or otherwise, does not necessarily constitute or imply its endorsement, recommendation, or favoring by the United States Government or any agency thereof. The views and opinions of authors expressed herein do not necessarily state or reflect those of the United States Government or any agency thereof.

\section{Distribution}

Approved for public release; further dissemination unlimited. 


\title{
OBSERVATION OF STIMULATED RAMAN SCATTERING IN AN OPTICAL FIBER AT THE FERMILAB A $\emptyset$ PHOTOINJECTOR
}

\author{
A.R. Fry, B. Taylor, M.J. Fitch, A.C. Melissinos \\ Department of Physics and Astronomy \\ University of Rochester \\ Rochester, NY 14627
}

\begin{abstract}
We have observed stimulated Raman scattering in a $2 \mathrm{~km}$ long optical fiber injected with an $81 \mathrm{MHz}$ train of $\sim 80$ ps pulses from a modelocked Nd:YLF oscillator operating at $\lambda=1054 \mathrm{~nm}$.
\end{abstract}


The $A \emptyset$ photoinjector at Fermilab is driven by a train of $800 \mathrm{UV}$ pulses $(\lambda=263 \mathrm{~nm})$ spaced at $1 \mathrm{MHz}$. These pulses are derived from a modelocked Nd:YLF oscillator $\lambda=1054 \mathrm{~nm}$, operating at $81 \mathrm{MHz}$ at a power level of $\sim 1 \mathrm{~W}$. In order to be able to compress the pulses in time the oscillator signal is coupled into a $2 \mathrm{~km}$ monomode optical fiber (Corning SMF-28, $8.3 \mu \mathrm{m}$ core diameter). Due to phase self-modulation the bandwidth is increased to $\Delta \lambda \sim 24 \AA$ and the pulse is chirped with $\Delta t \sim 130$ ps.

The operation of the oscillator is controlled primarily by adjusting the pump lamp current and the cavity length. Under normal conditions we seek stable amplitude and bandwidth at the output of the fiber. Spectral analysis of this output (using an HP 54600A optical spectrum analyzer) is shown in Fig. 1 for different current settings and shows clearly the Stokes line at a level of 40-50 db below the signal. The position of the line is in the range of the known frequency of the vibrational levels of $\mathrm{SiO}_{2}$. We find for the vibrational energy of fused silica

$$
\frac{\Delta \nu}{c}=\frac{\Delta \lambda}{\lambda_{2} \lambda_{1}}=428 \pm 5 \mathrm{~cm}^{-1}
$$

to be compared to a published value of $467 \mathrm{~cm}^{-1}$ for $\mathrm{SiO}_{2}[1]$.

We define the Raman yield as the ratio of the Stokes line to the pump power. The Raman yield is plotted as a function of the laser intensity entering the fiber in Fig. 2. A clear exponential growth is observed as expected for stimulated Raman scattering [2]. To obtain the laser intensity from the optical analyzer amplitude we note that at the normal operating current of $25 \mathrm{~A}$ the laser power is $P=0.8 \mathrm{~W}$ in the $f=81 \mathrm{MHz}$ pulse train, and the pulse width $\tau=80 \mathrm{ps}$; furthermore the coupling efficiency into the fiber is $\eta=0.42$ and the fiber area $A=54 \mu \mathrm{m}^{2}$. Thus

$$
I=(25 A)=\eta \frac{P}{\tau \cdot f \cdot A}=96 \mathrm{MW} / \mathrm{cm}^{2}
$$

The data are summarized in Table I.

\section{$\underline{\text { Table I }}$}

Lamp Current Intensity in Fiber Raman Yield

$\begin{array}{ccc}(A) & \mathrm{MW} / \mathrm{cm}^{2} & \left(P_{S} / P_{P}\right) \\ 25 & 96 & 3.0 \times 10^{-6} \\ 26 & 103 & 4.4 \times 10^{-6} \\ 27 & 120 & 10.7 \times 10^{-6} \\ 28 & 164 & 132.0 \times 10^{-6} \\ 29 & 152 & 66.7 \times 10^{-6}\end{array}$


For a stimulated process

$$
P_{S}=P_{P} e^{g_{s} \ell_{\mathrm{eff}}}
$$

where $P_{S}, P_{P}$ are the power in the Stokes line and in the pump, $g_{s}$ the gain coefficient and $\ell_{\text {eff }}$ the effective length for stimulated emission. By writing the exponential gain in the form

$$
g_{s} \ell_{\mathrm{eff}}=\left(g_{s} / I\right) \ell_{\mathrm{eff}} I
$$

we obtain from Fig. 2

$$
\left(g_{s} / I\right) \ell_{\mathrm{eff}}=56 \times 10^{-3} \mathrm{~cm}^{2} / \mathrm{MW}
$$

The value of $\left(g_{s} / I\right)$ for $\alpha$-quartz is given in ref. [3] as $\left(g_{s} / I\right)=0.5 \times 10^{-3} \mathrm{~cm} / \mathrm{MW}$; adopting this value for the material of the fiber, yields

$$
\ell_{\mathrm{eff}} \simeq 78 \mathrm{~cm}
$$

This result is consistent with the short pulse duration. Since the pump and Stokes radiation have different velocities of propagation in the fiber, the two pulses overlap only over a limited path length and the phase relation between the pump and Stokes fields is not constant. We also have not accounted for the initial spontaneous emission of the Stokes line.

As the pump intensity is increased the Stokes line continues to grow and becomes quite distinct as shown in Fig. 3a. The corresponding frequency spectrum of the pump at the exit of the fiber is shown in Fig. 3b. If the laser cavity is detuned, the operation of the laser becomes unstable and intense "spikes" of short pulses appear. Under these conditions the Stokes line can almost reach the pump level as seen in Fig. 3c. Furthermore the $n=2, n=3$ Stokes and the anti-Stokes lines become visible. It is evident that small instabilities in laser operation can give rise to large fluctuations in the output of the fiber and should be avoided during normal operation.

We thank Dr. Helen Edwards for her effective leadership of the A $\emptyset$ photoinjector project and Mr. Ralph Pasquinelli for the loan of the optical spectrum analyzer. This work was supported in part by Dept. of Energy Grant DE-FG02-91ER40685. 


\section{$\underline{\text { References and Notes }}$}

1. A. Yariv, "Quantum Electronics", J. Wiley, New York, NY (1989) p.468.

2. Y.R. Shen and N. Bloembergen Phys. Rev. 137A, 1787 (1965); G. Eckhardt et al. Phys. Rev. Lett. 9, 455 (1962).

3. W.D. Johnston et al., App. Phys. Lett. 13, 190 (1968); R.G. Smith, Applied Optics 11, 2489 (1972).

\section{Figure Captions}

Fig. 1 The spectrum at the output of the fiber for different lamp currents. The Stokes line is clearly observed.

Fig. 2 Raman yield as a function of laser intensity at the fiber input shows exponential growth as expected for stimulated Raman scattering.

Fig. 3 (a) Spectrum at the fiber output for increased pump power but stable laser operation. (b) Detail of the spectrum at the fiber output at the pump frequency for the same operating conditions as in (a); note the large bandwidth. (c) By detuning the laser cavity, operation becomes unstable and the Stokes line reaches within $3.5 \mathrm{db}$ of the pump; note also the $n=2$ and $n=3$ Stokes lines and the anti-Stokes line. 
Raman Spectrum at Different Optical Input Powers (W)

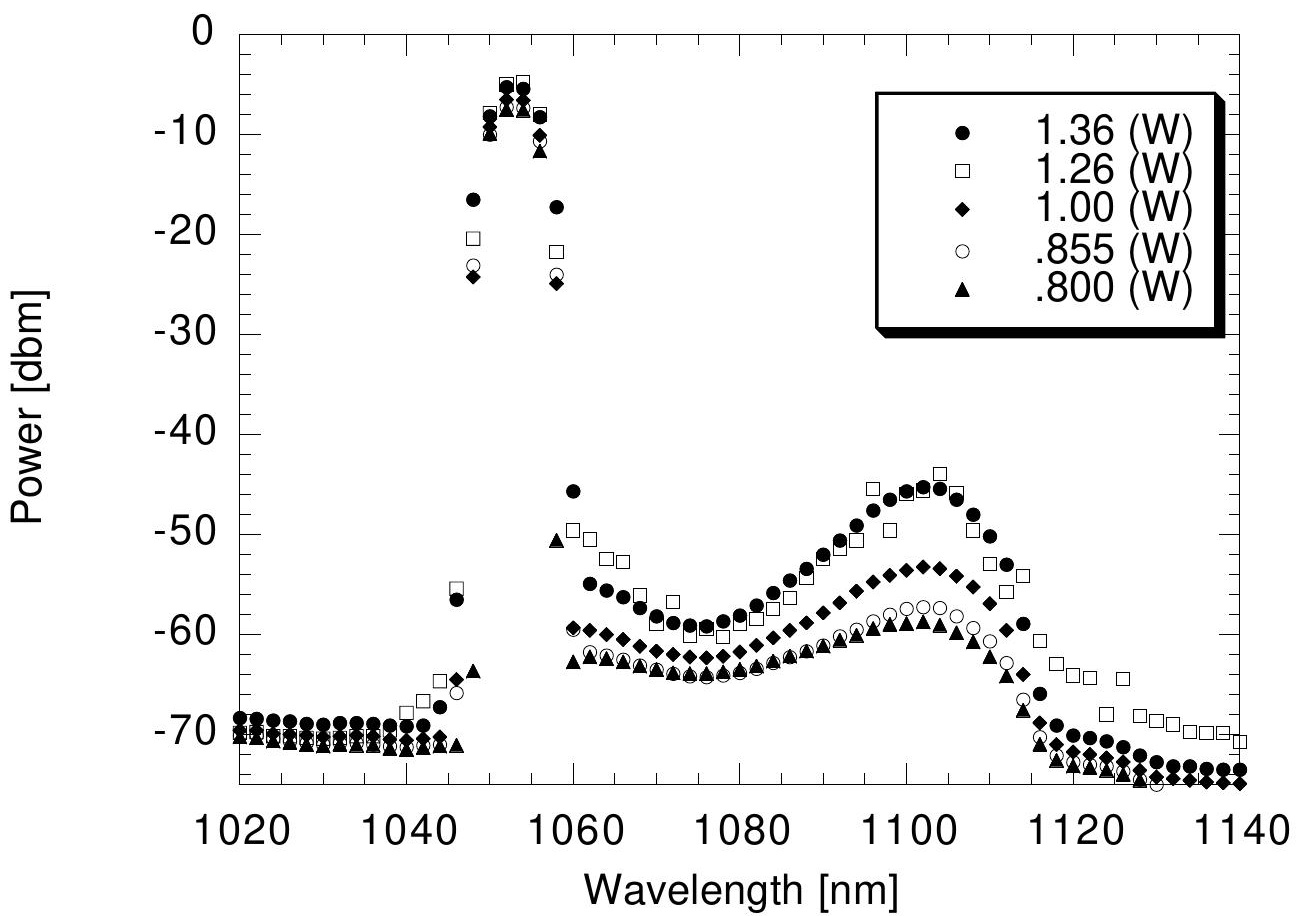


Power ratio between laser and first Raman line

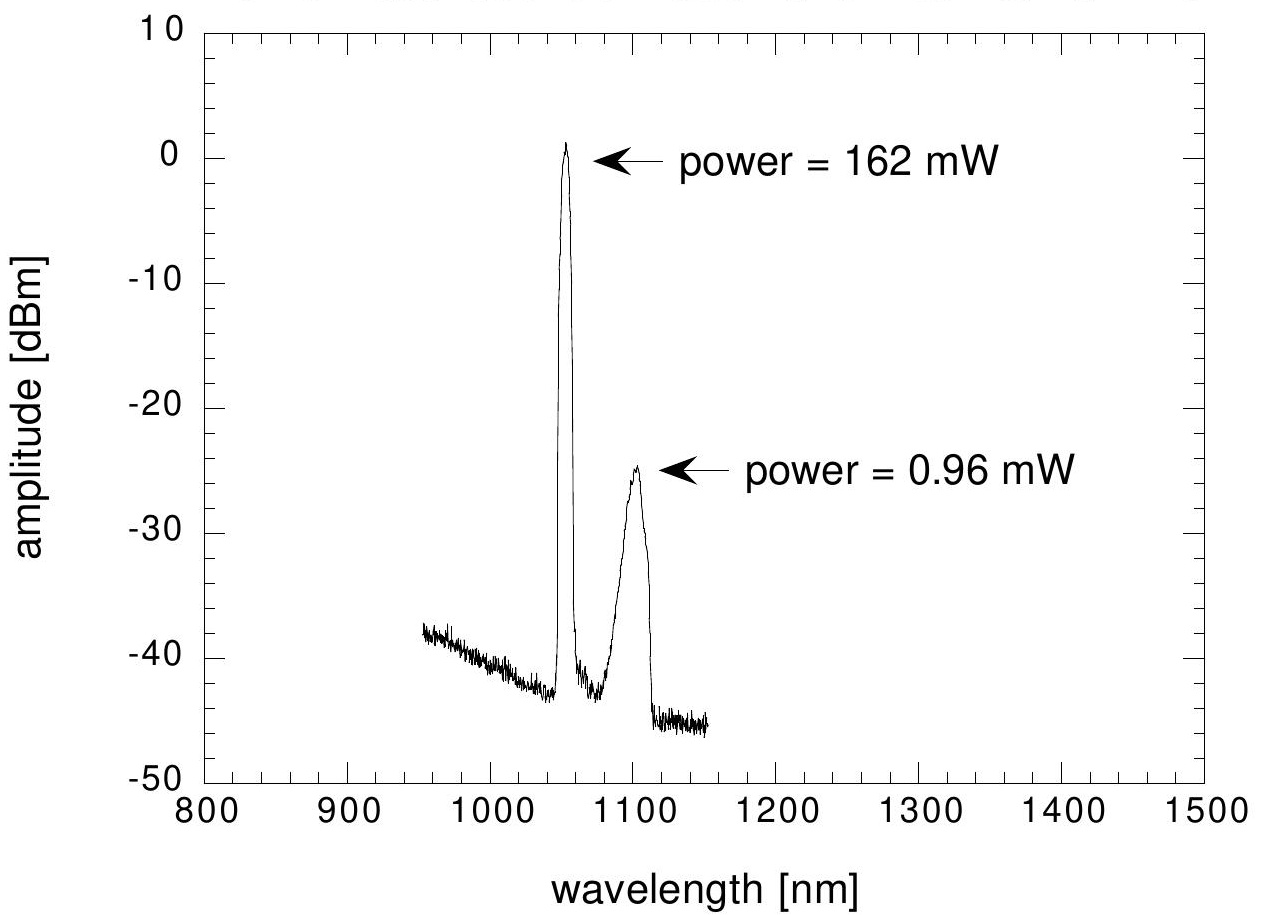


Central laser line spectral detail

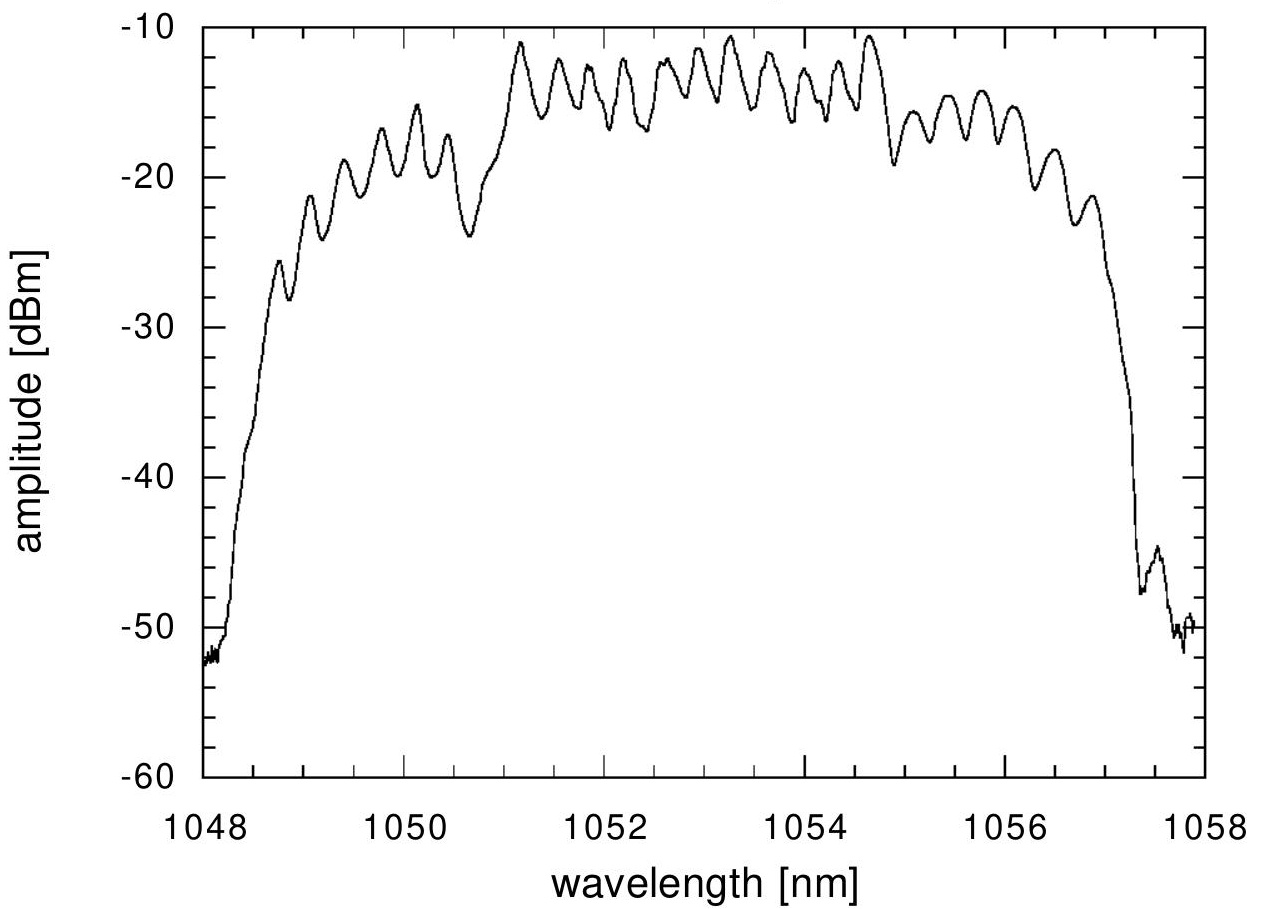


Multiple Raman lines from unstable laser

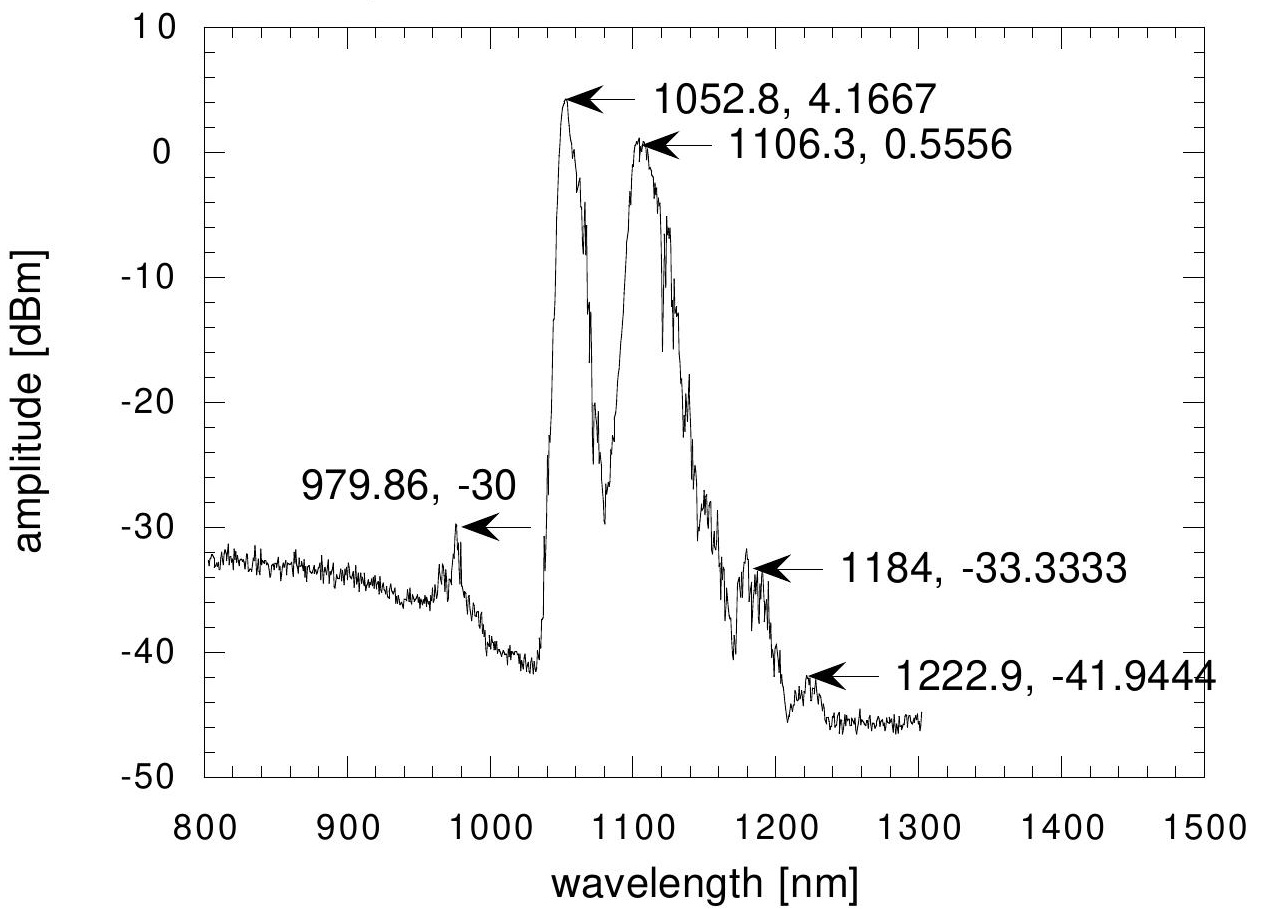

